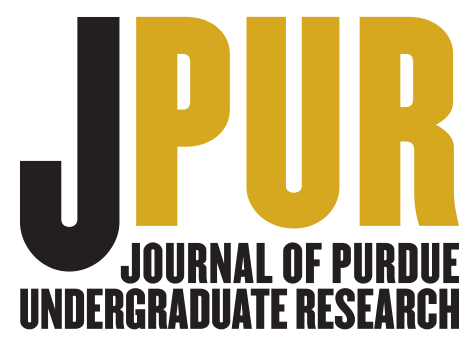

STEM

\section{Bioengineered Cell Niche for \\ Skeletal Muscle Regeneration}

\section{Student researcher: Nicole Whittern}

Skeletal muscles can self-repair minor strains, lacerations, and contusions; however, in cases of volumetric muscle loss and muscle degenerative diseases, tissue fails to regenerate. Current cellbased therapies, such as myoblast transplantation, have significant drawbacks of low survival rates and engraftment efficacy, mainly due to the absence of a supportive cell microenvironment. Scaffolds that mimic the natural cell microenvironment provide a robust platform to support cell adhesion, migration, proliferation, and differentiation. Electrospinning is a versatile technology platform used for fabricating the fiber scaffold that mimics the extracellular matrix. Thus, we aim to reconstitute the cell microenvironment through development of aligned fiber scaffolds by electrospinning as oriented muscle fibers create a natural microenvironment of myogenic cells. In particular, aligned fiber scaffolds will be optimized in terms of mechanical properties and fiber diameters while fiber curvature and mechanical stiffness provide significant physical cues for myogenic cell behaviors. Here, we fabricated and characterized electrospun polyester fiber scaffolds with different diameters from microscale to nanoscale. The mechanical properties of the fabricated nanofibers were found to be in the range of contractile muscles, as evidenced from atomic force microscopy measurements. With these scaffolds, $\mathrm{C} 2 \mathrm{C} 12$ myoblasts were seeded and analyzed for the initial attachment.

It was shown that aligned fibers with varying diameters resulted in different responses in cell attachment, indicating the role of cell topography sensing in cell-biomaterial interactions. Current ongoing studies focus on long-term in vitro culture of scaffolds in a custom-made muscle bioreactor emulating the contraction/ relaxation of skeletal muscle tissue.
Research advisor Meng Deng writes: "Nicole has demonstrated great professionalism with dedicated efforts in fabricating the polymer scaffolds and handling the cell cultures throughout the time she has participated in my lab. Additionally, she has demonstrated the ability to combine her knowledge of biological systems with mechanical applications through her assistance in the design of a bioreactor."

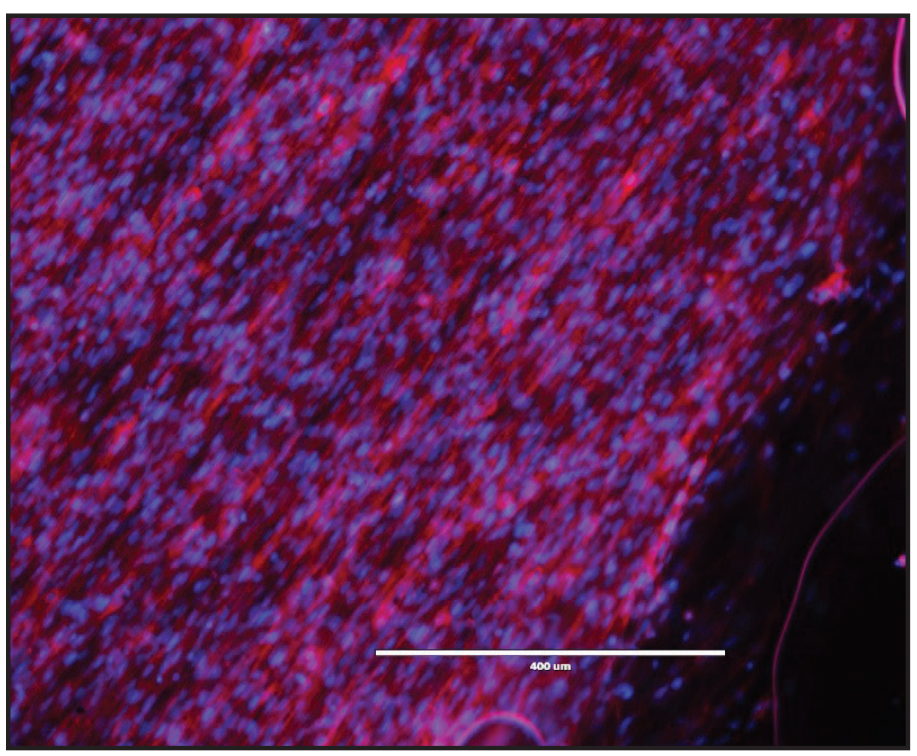

Fluorescence image of myoblasts on aligned fibers after 24 hours of cell seeding; cells spread along the direction of fiber orientation on aligned scaffolds indicating the effect of contact guidance. Red: cytoskeletal actin; blue: nuclei. 